\title{
Influence of Swelling and Diffusion-Induced Convection on Polymer Sorption Processes
}

\author{
Sacide Alsoy \\ Dept. of Chemical Engineering, Izmir Institute of Technology, Cankaya-Izmir, Turkey
}

J. L. Duda

The Pennsylvania State University, University Park, PA 16802

\begin{abstract}
Unsteady-state sorption of a vapor or liquid by a polymer is modeled to include the influence of the moving phase boundary associated with the polymer swelling and diffusion-induced convection. A formulation presented clearly elucidates the influence of these two effects on the overall sorption process. Numerical solutions of the model equations indicate the errors that can be induced when swelling or convection are neglected. For most polymer-solvent systems, the influence of the diffusion-induced convection associated with volume changes in mixing can be neglected in the analysis of sorption processes. In contrast, the correction for the moving boundary or swelling of the polymer phase can be quite significant, particularly when a large step change in solvent concentration is considered.
\end{abstract}

\section{Introduction}

The unsteady-state transport of low molecular weight substances in polymer solids and melts is an important phenomena in many polymer process operations, and in the utilization of polymers. Modeling of these processes, which involves the solution of an unsteady-state diffusion equation with associated boundary conditions, has been extensively investigated in numerous publications. It is generally recognized that the nonlinearity of the equations due to the concentration dependency of the molecular diffusivity is a complication associated with such polymer-solvent systems. However, there are other complications which are not included in most models of these polymer-solvent processes. When a vapor or liquid is sorbed or desorbed to any significant extent, the boundary at the interface between the polymer and the liquid or vapor moves. This moving boundary significantly complicates the analysis of the process in most conventional formulations. Secondly, convective flows in the polymer-solvent media can be induced by the mutual diffusion process. It is surprising that it has become a common practice to ignore these two complications without justification. In this work, both conventional formulation of the polymer sorption process in spatial (Eulerian) coordinates and a formulation derived by

Correspondence concerning this article should be addressed to J. L. Duda.
Duda and Vrentas (1971) in polymer material (Lagrangian) coordinates are presented in which the swelling and solventinduced convection are clearly delineated. The coordinate system transformation utilized by Duda and Vrentas results in an equation in the form of the conventional unsteady-state diffusion equation with an effective diffusivity. In this formalism, the effective diffusivity is a product of the conventional mutual binary diffusion coefficient with a second term which accounts for the influence of the moving boundary and the diffusion induced convection. This equation is then solved for a range of conditions to elucidate the influence of these two phenomena on the sorption process. These results can be used as a guide to future investigators to determine what errors are introduced when the moving boundary or the convection is ignored.

\section{Formulation of the Problem}

The following analysis considers sorption of a vapor or liquid into a solid or molten polymer slab or sheet. In the dimensionless formulation, the analysis is also applicable to desorption where the low molecular weight species is diffusing out of the polymer phase. The model equations were developed based on the geometry shown in Figure 1 where unsteady state (1-D) binary diffusion takes place in a thin film 


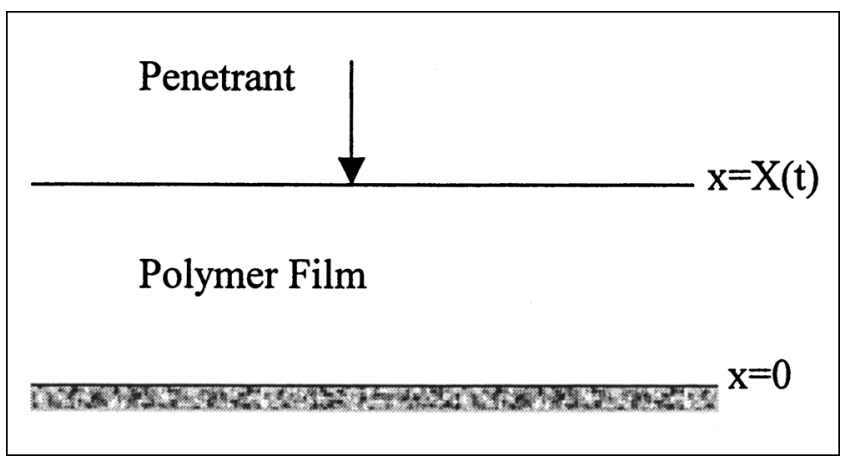

Figure 1. Geometry of sorption process.

sitting on an impermeable flat plate. Initially, the polymer film has a thickness L. At $t=0$, it is exposed to a penetrant that establishes its equilibrium concentration at the surface instantaneously. Other assumptions are: isothermal system, no reactions, negligible influence of pressure, and a Fickian diffusion process in which the relaxation of the polymer chains is fast compared to the rate of diffusion. Finally, to focus on the effects of the moving boundary and convection, the mutual binary diffusion coefficient is considered to be independent of concentration. In conventional analyses of most mass-transfer processes, the mass average velocity is used as a reference frame, and the formulation for the polymer sorption process results in three coupled equations for the mass fraction of the penetrant $\left(\omega_{1}\right)$, mass average velocity, $v$, and the position of the moving boundary X (Duda and Vrentas, 1968). Species continuity equation for the penetrant and total continuity equation can be written as follows

$$
\begin{array}{r}
\rho \frac{\partial \omega_{1}}{\partial t}+\rho v \frac{\partial \omega_{1}}{\partial x}-\frac{\partial}{\partial x}\left(\rho D \frac{\partial \omega_{1}}{\partial x}\right)=0 \\
\frac{\partial \rho}{\partial t}+v \frac{\partial \rho}{\partial x}+\rho \frac{\partial v}{\partial x}=0
\end{array}
$$

If Eq. 1 is multiplied by $\partial \rho / \partial \omega_{1}$, then

$$
\frac{\partial \rho}{\partial t}+v \frac{\partial \rho}{\partial x}=\frac{1}{\rho} \frac{\partial \rho}{\partial \omega_{1}} \frac{\partial}{\partial x}\left[\rho D \frac{\partial \omega_{1}}{\partial x}\right]
$$

Combining Eqs. 2 and 3 results in the following expression for the gradient of mass average velocity.

$$
\frac{\partial v}{\partial x}=-\frac{1}{\rho^{2}} \frac{d \rho}{d_{\omega_{1}}} \frac{\partial}{\partial x}\left(\rho D \frac{\partial \omega_{1}}{\partial x}\right)
$$

A jump balance for polymer at the moving phase boundary between the polymer solution and the vapor phase is

$$
\left(\rho_{2} v_{2}\right)^{p}-\rho_{2}^{p} \frac{d X}{d t}=\left(\rho_{2} v_{2}\right)^{v}-\rho_{2}^{v} \frac{d X}{d t}
$$

Inserting the definition of total flux $\rho_{2} v_{2}=\rho_{2} v+j_{2}$ and considering the fact that polymer is nonvolatile $\rho_{2}^{v}=0$, Eq. 5 then reduces to the following form

$$
\frac{d X}{d t}=v+\frac{j_{2}}{\rho_{2}}
$$

The utilization of a well-known result $j_{2}=-j_{1}$ and using the definition of mass flux of penetrant with respect to the mass average velocity $j_{1}=-\rho D\left(\partial \omega_{1} / \partial x\right)$ results in the following expression for the time dependence of film thickness

$$
\frac{d X}{d t}=\left.v\right|_{x=X(t)}+\left[\frac{D \frac{\partial \omega_{1}}{\partial x}}{1-\omega_{1}}\right]_{x=X(t)}
$$

In this coordinate system, the associated initial and boundary conditions are

$$
\begin{aligned}
\omega_{1}(x, 0)=\omega_{10} \quad \omega_{1}[X(t), t] & =\omega_{1 E} \\
\frac{\partial \omega_{1}}{\partial x}(0, t) & =0 \quad v(0, t)=0 X(0)=L
\end{aligned}
$$

The thermodynamics of the polymer-solvent systems for constant temperature and pressure conditions reduces to: $\rho=$ $\rho\left(\omega_{1}\right)$.

It is obvious from the above equations that the inclusion of the effects of the moving boundary and diffusion-induced convection greatly complicate the analysis of the sorption process when formulated in a conventional manner. In this formulation, the convection induced by the diffusion process (Eq. 4) can only be eliminated by assuming a constant density system. The complexity of this formulation has led to the common practice of neglecting the polymer swelling and convection in the analysis of polymer sorption processes, and very few solutions of this complete set of equations are available. Following a suggestion by Crank (1975), Duda and Vrentas (1971) were able to formulate this problem in a simpler form by utilizing different length and concentration variables and the mass average reference frame. In the following development, the Duda-Vrentas coordinate transformation is used in conjunction with defining the diffusion flux relative to the volume average velocity to provide a single equation formulation which reveals the role of these two processes.

The basic equations describing the sorption process are the species continuity equations for the solvent and polymer

$$
\begin{aligned}
& \frac{\partial \rho_{1}}{\partial t}+\frac{\partial\left(\rho_{1} v_{1}\right)}{\partial x}=0 \\
& \frac{\partial \rho_{2}}{\partial t}+\frac{\partial\left(\rho_{2} v_{2}\right)}{\partial x}=0
\end{aligned}
$$

Introducing a diffusive flux $j_{1}^{o}=\rho_{1}\left(v_{1}-v_{2}\right)$, a new concentration variable $q_{1}=\rho_{1} / \rho_{2} \hat{V}_{2}^{o}$, and a new length variable $\xi(x, t)=\int_{0}^{x} \rho_{2} \hat{V}_{2}^{0} d x$ yields the following equation to describe the sorption process

$$
\left(\frac{\partial q_{1}}{\partial t}\right)_{\xi}+\left(\frac{\partial j_{1}^{0}}{\partial \xi}\right)_{t}=0
$$


In terms of the new length and concentration variables, diffusive flux $j_{1}^{0}$ can be written as follows

$$
j_{1}^{0}=D\left(\rho_{2} \hat{V}_{2}^{0}\right)^{2}
$$

Substitution of Eq. 12 into Eq. 11 gives

$$
\left(\frac{\partial q_{1}}{\partial t}\right)_{\xi}=\frac{\partial}{\partial \xi}\left[D_{\text {eff }} \frac{\partial q_{1}}{\partial \xi}\right]
$$

where the effective diffusivity is defined by the following equation

$$
D_{\text {eff }}=D\left(\rho_{2} \hat{V}_{2}^{0}\right)^{2}
$$

Consequently, the formulation of the sorption process in the new coordinate system is a partial differential equation which has a standard form of the species continuity equation with an effective diffusivity which is a function of concentration. As defined by Eq. 14, the effective diffusivity is the product of two terms. The first term is the conventional mutual binary diffusion coefficient, which is assumed to be constant and the second term accounts for the influence of the moving boundary and the diffusion induced convection on the overall sorption process. It should be emphasized that this formulation is independent of the volumetric behavior of the system. The volumetric behavior of a system will dictate the concentration dependency of this effective diffusivity. This concentration dependency will be different for a system exhibiting a volume change on mixing compared to a system for which the partial molar volumes are constant.

Equation 13 is a nonlinear equation which is subject to the following initial and boundary conditions for the sorption process

$$
\begin{aligned}
&\left(\frac{\partial q_{1}}{\partial \xi}\right)_{\xi=0}=0 \quad q_{1}\left(\xi_{L}, t\right)=q_{1 E} \\
& q_{1}(\xi, 0)=q_{1 o} \quad \xi_{L}=\rho_{2 o} \hat{V}_{2}^{o} L
\end{aligned}
$$

This formulation can be put into a dimensionless form utilizing the following dimensionless variables, where reference diffusivity $D_{o}$ is chosen as the effective diffusivity calculated at the initial composition of the mixture. The dimensionless formulation of the sorption process is given by

$$
\begin{aligned}
q_{1}^{*} & =\frac{q_{1}-q_{1 o}}{q_{1 E}-q_{1 o}} \quad \xi^{*}=\frac{\xi}{\xi_{L}} \quad t^{*}=\frac{t D_{o}}{\xi_{L}^{2}} \\
\frac{\partial q_{1}^{*}}{\partial t^{*}} & =\frac{\partial}{\partial \xi^{*}}\left[\frac{D_{\text {eff }}}{D_{o}} \frac{\partial q_{1}^{*}}{\partial \xi^{*}}\right] \\
\left(\frac{\partial q_{1}^{*}}{\partial \xi^{*}}\right)_{\xi^{*}=0} & =0 \quad q_{1}^{*}\left(1, t^{*}\right)=1 \quad q_{1}^{*}\left(\xi^{*}, 0\right)=0
\end{aligned}
$$

To elucidate the influence of the moving boundary and diffusion-induced convection on the sorption process, Eq. 17 with its associated boundary conditions was solved numerically for a range of conditions. As Eq. 14 indicates, the influence of the moving boundary and the convection on the diffusion process is directly related to the polymer concentration and the specific volume of the polymer. For illustrative purposes, the specific volume of the nonideal mixture is considered to be represented by a second-order polynomial

$$
\hat{V}=a_{o}+a_{1} \omega_{1}+a_{2} \omega_{1}^{2}
$$

The number of coefficients in this equation can be reduced by using the following limits

$$
\begin{gathered}
\omega_{1}=0 \Rightarrow \hat{V}=\hat{V}_{2}^{o} \\
\omega_{1}=1 \Rightarrow \hat{V}=\hat{V}_{1}^{o}
\end{gathered}
$$

Finally, the specific volume of the mixture can be given by Eq. 22

$$
\hat{V}=\hat{V}_{2}^{o}+a_{1} \omega_{1}+\left\lfloor\left(\hat{V}_{1}^{o}-\hat{V}_{2}^{o}\right)-a_{1}\right\rfloor \omega_{1}^{2}
$$

The weight fraction of the solvent $\omega_{1}$ is related to the new concentration variable $q_{1}$ as follows

$$
\omega_{1}=\frac{q_{1} \hat{V}_{2}^{o}}{1+q_{1} \hat{V}_{2}^{o}}
$$

The partial specific volumes of the components are obtained by using the following equation

$$
\bar{V}_{i}=\left(\frac{\partial V_{m}}{\partial m_{i}}\right)_{T, P, m_{j}}
$$

which also can be written as follows

$$
\bar{V}_{i}=\hat{V}+\sum m_{i}\left(\frac{\partial \hat{V}}{\partial m_{i}}\right)_{T, P, m_{j}}
$$

When the effect of volume change on mixing is negligible, then the partial specific volume of each component at all concentrations of the mixture becomes equal to that of the pure component and the specific volume of the mixture is given by the following equation

$$
\hat{V}=\hat{V}_{2}^{o}+\omega_{1}\left(\hat{V}_{1}^{o}-\hat{V}_{2}^{o}\right)
$$

The relative deviation from the ideal volumetric behavior can then be expressed as follows

$$
\% \text { Deviation }=\left(\frac{\hat{V}_{\text {nonideal }}-\hat{V}_{\text {ideal }}}{\hat{V}_{\text {ideal }}}\right) \times 100
$$

Substitution of Eqs. 22 and 26 into Eq. 27 gives

$$
\% \text { Deviation }=\left(\frac{\omega_{1} \omega_{2}\left[a_{1}-\left(\hat{V}_{1}^{o}-\hat{V}_{2}^{o}\right)\right]}{\hat{V}_{2}+\omega_{1}\left(\hat{V}_{1}^{o}-\hat{V}_{2}^{o}\right)}\right) \times 100
$$


The definition of effective diffusivity, given by Eq. 14, can be rewritten by considering its concentration dependency in an explicit manner as follows

$$
D_{\text {eff }}=D\left[\left(1-\omega_{1}\right) \frac{\hat{V}_{2}^{o}}{\hat{V}}\right]^{2}
$$

where $\hat{V}$ is defined by Eqs. 22 and 26 for nonideal and ideal mixtures, respectively.

\section{Results and Discussion}

In the formulation presented above, the effective diffusivity results from the moving boundary, the convective flows induced by diffusion, as well as the conventional, mutual binary diffusion coefficient. This equation cannot be solved analytically since it is a nonlinear equation, even when the binary mutual diffusion coefficient is not a function of concentration. The nonlinearity of diffusion equation is reflected by concentration dependency of both mass density of the polymer $\rho_{2}$ and the specific volume of the mixture. To illustrate the influence of the moving boundary and convection on the sorption process, Eqs. 17-18 were solved using an implicit finite difference technique with a variable grid so that a finer finite difference grid spacing was imposed near the interface where steep concentration gradients occur (Alsoy and Duda, 1998). This numerical solution gives the concentration of the solvent or penetrant in the polymer as a function of position and time. In most practical applications, the total amount of solvent in the polymer is desired as a function of time. In dimensionless form, this is referred to as the sorption uptake curve or fractional approach to equilibrium as defined by $M$ $=\left[\left(M_{t}-M_{i}\right) /\left(M_{\infty}-M_{i}\right)\right]$ where $M_{t}$ is the mass of penetrant in the polymer at any time, $M_{\infty}$ is the final equilibrium mass uptake, and $M_{i}$ is the initial solvent mass in the polymer phase. Three cases for dimensionless mass uptake are considered:

- $M_{0}$ is the fractional approach to equilibrium without considering the moving boundary or convection. For constant $D$, this results from the well-known analytical solution (Crank, 1975).

- $M_{1}$ is the fractional approach to equilibrium from Eqs. 17 and 18 which includes only the contribution of swelling, where the specific volume of the mixture is calculated from Eq. 26 and, consequently, the volume average velocity is zero.

- $M_{2}$ is the fractional approach to equilibrium from Eqs. 17 and 18 which includes both the moving boundary and convection contributions, where the specific volume of the mixture is calculated from Eq. 22.

In these calculations, the specific volume of pure components $\hat{V}_{1}^{o}, \hat{V}_{2}^{o}$ were chosen as $1.15 \mathrm{cc} / \mathrm{g}$ and $0.9 \mathrm{cc} / \mathrm{g}$, respectively, which are typical values for many polymer solvent systems. The parameter $a_{1}$ in Eq. 28 was adjusted to control the maximum deviation in the volume change due to mixing which was chosen based on the experimental data reported in the literature. \% relative deviations from ideal mixing, chosen for simulations, and corresponding $a_{1}$ values calculated from Eq. 28 are given in Table 1.

To illustrate the influence of the moving boundary or swelling process, as well as the convection on the overall
Table 1. \% Deviation from Ideal Volumetric Behavior, Initial and Equilibrium Weight Fractions of the Solvent and $a_{1}$ Values Used in the Simulations.*

\begin{tabular}{cccc}
\hline $\begin{array}{c}\% \text { Dev. from } \\
\text { Ideal Mixing }\end{array}$ & $\omega_{1 o}$ & $\omega_{1 E}$ & $a_{1}$ \\
\hline $1 \%$ & 0 & 0.1 & 0.353 \\
$-1 \%$ & 0 & 0.1 & 0.147 \\
$1 \%$ & 0 & 0.2 & 0.309 \\
$-1 \%$ & 0 & 0.2 & 0.191 \\
$2 \%$ & 0 & 0.2 & 0.369 \\
$-2 \%$ & 0 & 0.2 & 0.131 \\
$4 \%$ & 0 & 0.2 & 0.488 \\
$-4 \%$ & 0 & 0.2 & 0.0125 \\
\hline
\end{tabular}

$* \hat{V}_{1}^{o}=1.15 \mathrm{cc} / \mathrm{g}, \hat{V}_{2}^{o}=0.9 \mathrm{cc} / \mathrm{g}$.

sorption process, the results of this study are presented in terms of a ratio of the mass uptake which includes the swelling and/or convection contributions to the mass uptake that would be realized when these effects are neglected, $M_{1} / M_{0}$ or $\mathrm{M}_{2} / \mathrm{M}_{1}$. It should be emphasized that for all cases the dimensionless time is based on the initial thickness of the polymer solution. The influence of the moving boundary or the polymer swelling on the sorption process is illustrated in Figures 2 and 3 . It is obvious that the ratio $\mathrm{M}_{1} / \mathrm{M}_{0}$ will approach one as the change in the weight fraction of solvent in the polymer during the sorption process approaches zero. Figure 2 shows a series of sorption processes in which the initial concentration of solvent in the polymer sample is zero, and the final equilibrium weight fraction of solvent is increased. As expected, swelling of the polymer reduces the rate at which the solvent is absorbed, and the effect is more significant for the larger step changes in solvent concentration.

Figure 3 illustrates that the effect of swelling not only depends upon the magnitude of the jump in solvent concentration, but also as a function of the solvent concentration at the beginning of the sorption process. These figures can be used

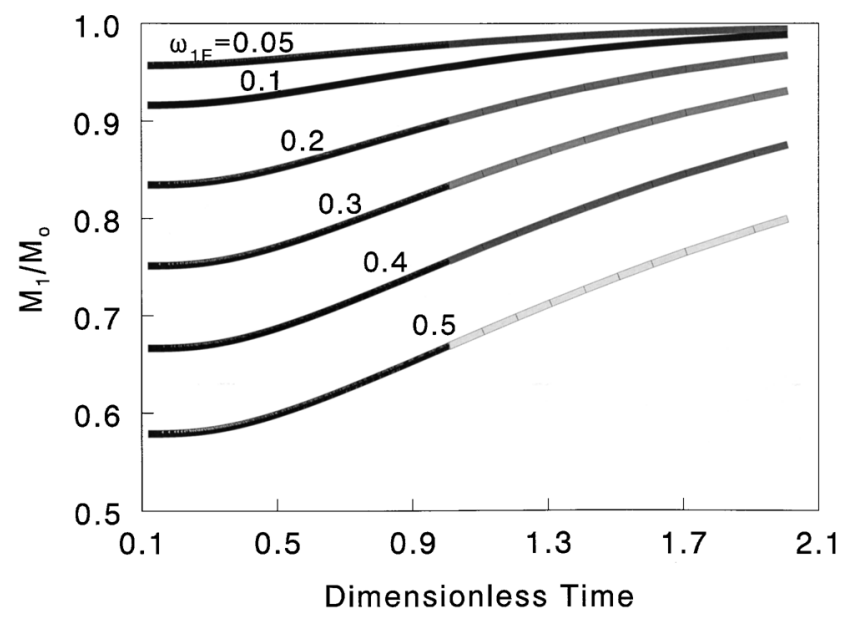

Figure 2. Effect of swelling on sorption uptake; initial concentration is zero, $\omega_{10}=0$.

The numbers on each curve represent equilibrium concentration. 


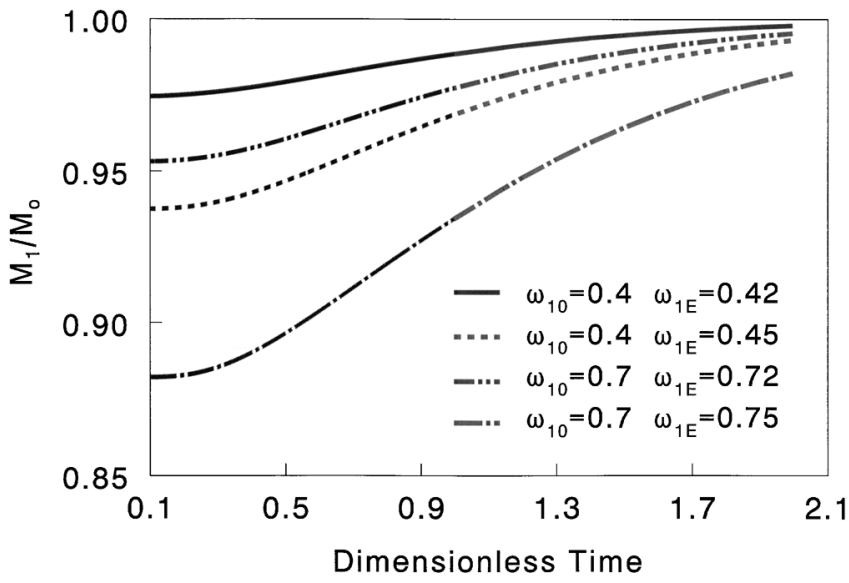

Figure 3. Effect of swelling on sorption uptake for a range of initial concentrations and two step sizes.

to estimate the relative error in the uptake curve, defined as $\left[1-\left(M_{1} / M_{o}\right)\right] x 100$, which will occur if the moving boundary or swelling of the polymer sample is neglected in the analysis. Furthermore, simulation results can be utilized to estimate the error that would occur if one simply evaluates the diffusion coefficient using the traditional formulas derived from analytical solution of the sorption equation (Crank, 1975). The initial slope method is one of the most commonly used one in which the value of the diffusion coefficient is deduced from an observation of the initial gradient of a graph of $M_{t} / M_{\infty}$ as a function of $\sqrt{t}$. In the half time method, diffusivity is calculated from

$$
D=\frac{0.1968}{\left(t / L^{2}\right)_{0.5}}
$$

in which $\mathrm{L}$ is the thickness of the polymer sheet, assumed constant, whose upper surface is exposed to the penetrant while its lower surface is impermeable, as shown in Figure 1. Thus, according to Eq. 30, if the swelling of the polymer sheet is negligible, then dimensionless time defined as $t^{*}=D t / L^{2}$ should be equal to 0.1968 , when $M_{t} / M_{\infty}$ is equal to 0.5 . It can be seen that dimensionless time used in this work can be also written as $t^{*}=D t / L^{2}$ if definitions of reference diffusivity $D_{o}$ $=D\left(\rho_{2 o} \hat{V}_{2}^{o}\right)^{2}$ and $\xi_{L}=\rho_{2 o} \hat{V}_{2}^{o} L$ are inserted into Eq. 16. Consequently, the magnitude of the error associated with the use of the half time method can be calculated as follows by comparing the dimensionless time obtained from the simulation results when $M_{t} / M_{\infty}=0.5, t_{0.5}^{*}$, with the corresponding value from the analytical solution, that is, 0.1968

$$
\% \text { Relative Error in } D=\left(\frac{0.1968-t_{0.5}^{*}}{0.1968}\right) \times 100
$$

As an illustration, the errors calculated from Eq. 31 are shown in Figure 4 for a case in which polymer is initially free of penetrant. As expected, the error in estimating the diffusivities from the half time method becomes significant if step size $\omega_{1 E}-\omega_{10}$ is above 0.05 due to the increasing effect of swelling.

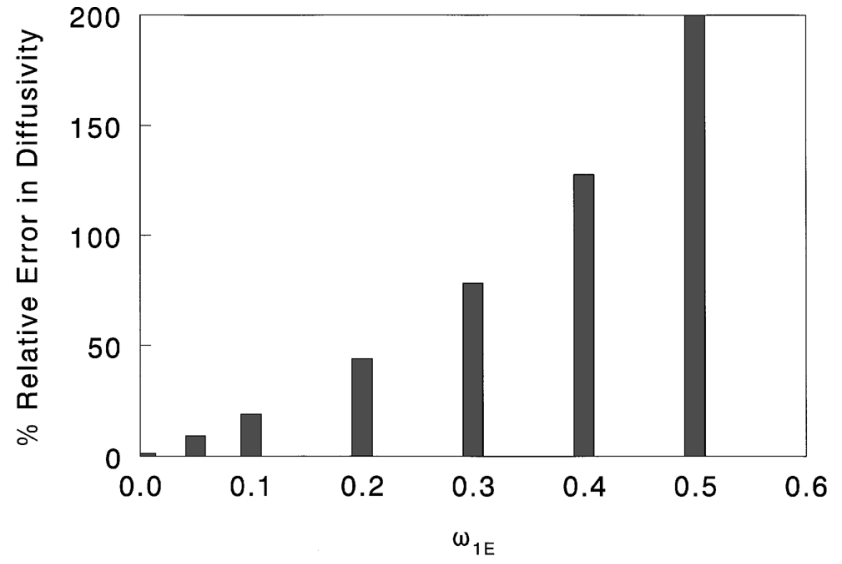

Figure 4. \% Relative error in estimating the diffusivities from the half time method as a function of equilibrium concentration, $\omega_{10}=0$.

The effect of volume change on mixing on the mass uptake process is shown in Figures 5 and 6. In all cases, the initial concentration of solvent was zero, and these two figures show the influence of the size of the step change, as well as the deviation from ideal volume behavior on the sorption process. Results are presented for positive and negative deviations from ideal volumetric behavior up to the maximum deviation of $4 \%$. However, most polymer-solvent systems do not show large deviations from ideal volumetric behavior, and the absolute value is typically 1-2\% or less (Wolf and Wendorff, 1990; Sasahara and Uedaira, 1994; Eichinger and Flory, 1968a,b). However, deviations over $5 \%$ from the ideal volume behavior have been reported for mixtures of polymers and supercritical fluids such as carbon dioxide (Beckman et al., 1990) and glassy polymer solvent systems (Maeda and Paul, 1987a,b). The results presented in Figures 5 and 6 indicate that when both the swelling and diffusion induced convection effects are included, the swelling effect dominates. Figure 7 illustrates the effect of concentration step size $\Delta \omega_{1}$ with $\omega_{1 o}$ $=0$ on the sorption uptake curve at different times during the process. The deviation from Crank's analytical solution is

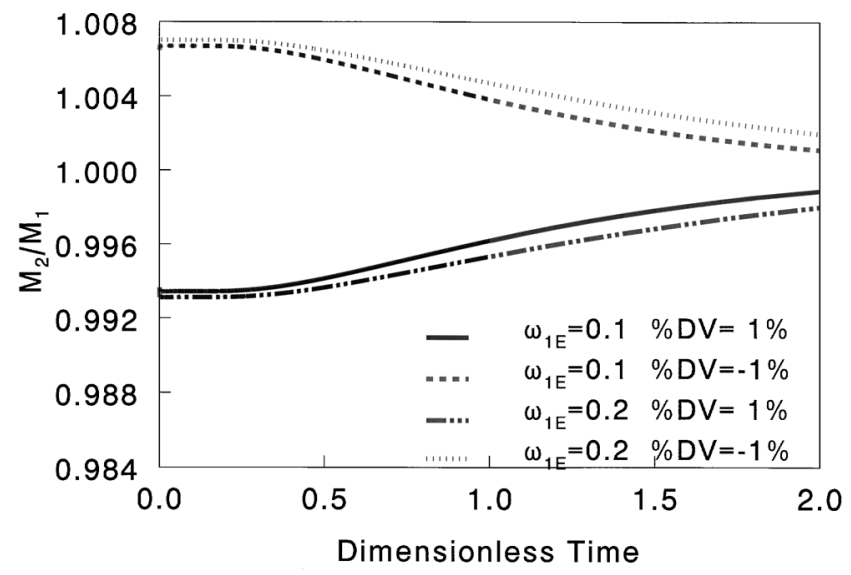

Figure 5. Effect of volume change on mixing on sorption uptake as a function of equilibrium concentration, $\omega_{10}=0$. 


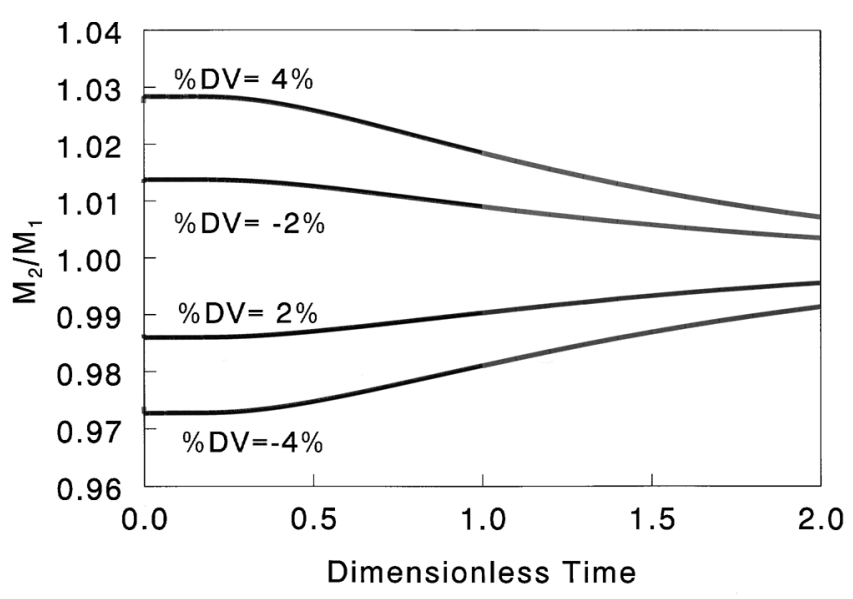

Figure 6. Effect of volume change on mixing on sorption uptake as a function of maximum \% volume change on mixing, $\% \mathrm{DV}, \omega_{10}=0, \omega_{1 E}=$ 0.2 .

negligible as the concentration step size is very small. This result is verified by the following equation in which in the limit of small step changes in the solvent concentration $\varphi_{1 E}$ $-\varphi_{10} \rightarrow 0 M_{1}$ approaches to $M_{o}$.

$$
\left[\frac{1-M_{o}}{1-M_{1}}\right]=\exp \left[\frac{D \pi^{2} t}{L^{2}}\left(\frac{\phi_{10}^{2}-\phi_{1 E}^{2}}{\phi_{10}^{2}}\right)\right]
$$

In the new coordinate system used here, the formulation of the sorption process results in the standard form of the species continuity equation with an effective diffusivity which accounts for the influence of the swelling and the diffusion induced convection on the overall sorption process. As defined by Eq. 29, the contribution of each effect depends upon the step size $\omega_{1 E}-\omega_{1 o} \%$ relative deviation from the ideal volumetric behavior and the specific volume of the components $\hat{V}_{1}^{o}, \hat{V}_{2}^{o}$. The effect of the first two parameters were analyzed for $\hat{V}_{1}^{o}=1.15 \mathrm{cc} / \mathrm{g}$ and $\hat{V}_{2}^{o}=0.9 \mathrm{cc} / \mathrm{g}$. To investigate the influence of specific volumes of components on uptake curves,

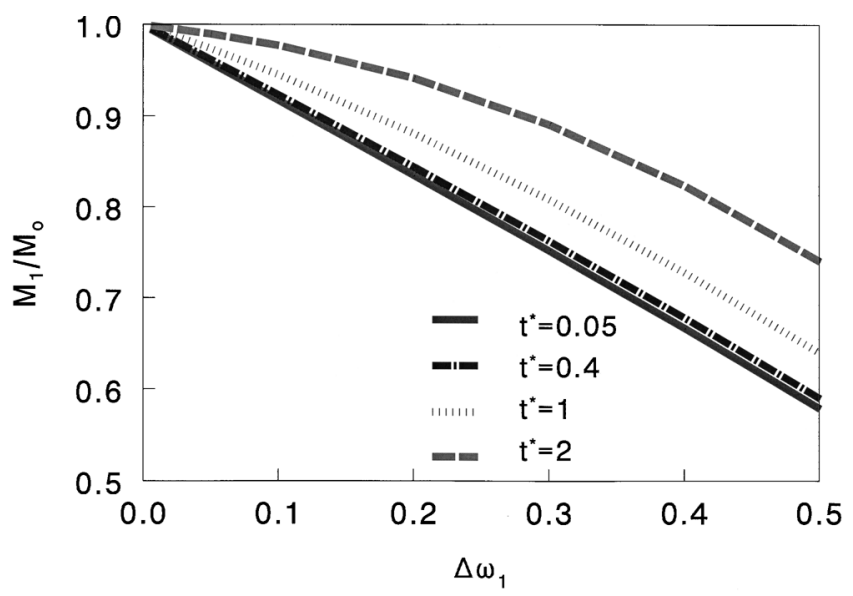

Figure 7. Effect of step size on sorption uptake, $\omega_{10}=0$.
Table 2. Effect of Specific Volume of Components on \% Relative Error in Diffusivities Estimated from the Half Time Method.*

\begin{tabular}{ccc}
\hline$\hat{V}_{1}^{o}\left(\mathrm{~cm}^{3} / \mathrm{g}\right)$ & $\hat{V}_{2}^{o}\left(\mathrm{~cm}^{3} / \mathrm{g}\right)$ & $\%$ Rel. Error in Diff. \\
\hline 0.6 & 0.6 & 13.2 \\
0.6 & 0.9 & 8.6 \\
0.6 & 1.2 & 7.4 \\
0.9 & 0.6 & 18.4 \\
0.9 & 0.9 & 13.2 \\
0.9 & 1.2 & 9.8 \\
1.2 & 0.6 & 22.9 \\
1.2 & 0.9 & 16.4 \\
1.2 & 1.2 & 13.2 \\
\hline
\end{tabular}

${ }^{*} \omega_{1 o}=0, \omega_{1 E}=0.1$.

simulations were carried out for a fixed step size $\omega_{1 E}-\omega_{1 o}$ $=0.1$ and different combinations of $\hat{V}_{1}^{o}$ and $\hat{V}_{2}^{o}$. The results are presented in Table 2 in terms of $\%$ relative error in diffusivities calculated from the half time method. It is obvious from these results that, for a given solvent $\left(\hat{V}_{1}^{o}=\right.$ Constant), the error in diffusivities decreases as the specific volume of polymer increases. In other words, for a fixed step size, the swelling effect is larger in polymer solvent mixtures in which the specific volume of the solvent is much higher than that of the polymer.

\section{Conclusion}

This study indicates that, for most polymer solvent systems, the influence of the diffusion-induced convection associated with volume changes on mixing can be neglected in the analysis of sorption processes. The correction term for the volume change on mixing is usually small and is negligible for most practical cases if the volume change on mixing is below $3 \%$. In contrast, the correction for the moving boundary or swelling of the polymer phase can be quite significant, particularly when a large range in concentration is covered in the sorption process. As stated earlier, the results shown here are based on a constant diffusivity. However, any specific form of the diffusivity-concentration relationship can be easily incorporated into this analysis, and these results can serve as a good approximate guide as to what kinds of errors are induced when the polymer swelling is neglected in the analysis of the process.

\section{Notation}

$a_{1}=$ polynomial coefficient

$D=$ binary diffusion coefficient

$D_{\text {eff }}=$ effective diffusivity defined by Eq. 9

$j_{1}^{o}=$ mass diffusion flux of solvent relative to velocity of polymer

$L=$ initial thickness of the polymer film

$m_{i}=$ mass of component $i$ in the mixture

$q_{1}=$ concentration variable

$t=$ time

$v=$ mass average velocity

$v_{i}=$ velocity of component $i$

$V_{m}=$ volume of the mixture

$\hat{V}=$ specific volume of the mixture

$\hat{V}_{i}^{o}=$ specific volume of pure component $i$

$\bar{V}_{i}=$ partial specific volume of component $i$ in the mixture

$\omega_{1}=$ weight fraction of the solvent

$x=$ distance variable in the direction of diffusion 
$X(t)=$ thickness of the polymer film at any time $t$ $\rho=$ density of the mixture

$\rho_{i}=$ mass density of component $i$ in the film

$\xi=$ length variable.

$\xi_{L}=$ quantity defined as $\xi_{L}=\rho_{2 o} \hat{V}_{2}^{o} L$

$\phi_{20}=$ initial volume fraction of the polymer

\section{Subscripts}

$o=$ initial value of concentration

$E=$ equilibrium value of concentration

\section{Literature Cited}

Alsoy, S., and J. L. Duda, "Drying of Solvent Coated Polymer Films," Drying Technol. J., 16, 15 (1998)

Beckman, E. J., R. Koningsveld, and R. S. Porter, "Mean-Field Lattice Equations of State. Modeling the Phase Behavior of Supercritical Gas-Polymer Mixtures," Macromol., 23, 2321 (1990).

Crank, J., The Mathematics of Diffusion, 2nd ed., Clarendon Press, Oxford (1975).

Duda, J. L., and J. S. Vrentas, "Diffusion in Atactic Polystyrene Above the Glass Transition Point," J. Poly. Sci., 6, 675 (1968).
Duda, J. L., and J. S. Vrentas, "Mathematical Analysis of Sorption Experiments," AIChE J., 17, 464 (1971).

Eichinger, B. E., and P. J. Flory, "Thermodynamics of Polymer Solutions: 1. Natural Rubber and Benzene," J. Trans. Faraday Soc., 64, 2035 (1968a).

Eichinger, B. E., and P. J. Flory, "Thermodynamics of Polymer Solutions: 3. Polyisobutylene and Cyclohexane," J. Trans. Faraday Soc., 64, 2061 (1968b)

Maeda, Y., and D. R. Paul, "Effect of Antiplasticization on Gas Sorption and Transport: I. Polysulfone," J. Poly. Sci., Poly. Phys., 25, 957 (1987a)

Maeda, Y., and D. R. Paul, "Effect of Antiplasticization on Gas Sorption and Transport: II. Poly (phenylene Oxide)," J. Poly. Sci., Poly. Phys., 25, 981 (1987b).

Sasahara, K., and H. Uedaira, "Volume and Compressibility Changes on Mixing Aqueous Solutions of the Amino Acid and Poly (ethylene Glycol)," Colloid \& Poly. Sci., 272, 385 (1994).

Wolf, M., and J. H. Wendorff, "Modified Equation of State Treatment For Volume Changes of Mixing," Poly. Commun., 31, 226 (1990).

Manuscript received Aug. 3, 2001, and revision received Feb. 7, 2002. 